\title{
Mathematical modelling of mantle convection at a high Rayleigh number with variable viscosity and viscous dissipation
}

\author{
Sumaiya B. Islam, Suraiya A. Shefa and Tania S. Khaleque*
}

\section{*Correspondence:}

tania.khaleque@du.ac.bd

Department of Applied

Mathematics, University

of Dhaka, Dhaka 1000,

Bangladesh

\begin{abstract}
In this paper, the classical Rayleigh-Bénard convection model is considered and solved numerically for extremely large viscosity variations (i.e., up to $10^{30}$ ) across the mantle at a high Rayleigh number. The Arrhenius form of viscosity is defined as a cut-off viscosity function. The effects of viscosity variation and viscous dissipation on convection with temperature-dependent viscosity and also temperature- and pressure-dependent viscosity are shown through the figures of temperature profiles and streamline contours. The values of Nusselt number and root mean square velocity indicate that the convection becomes significantly weak as viscosity variation and viscous dissipation are increased at a fixed pressure dependence parameter.
\end{abstract}

Keywords: Mantle convection, Variable viscosity, Viscous dissipation, Rayleigh-Bénard convection, Viscosity variation

Mathematics Subject Classification: 76D05, 80A19, 8610

\section{Introduction}

Convection in mantle is responsible for most of the physical and chemical phenomena happening on the surface and in the interior of the Earth, and it is caused by the heat transfer from the interior to the Earth's surface. Even though there are some debates, it is quite well established that convection in the mantle is the driving mechanism for plate tectonics, seafloor spreading, volcanic eruptions, earthquakes, etc. [1]. However, the mechanism of mantle convection is still an unsolved mystery since the rheology of mantle rocks is extremely complicated [2-4]. Temperature, pressure, stress, radiogenic elements, creep, and many other factors influence the mantle's behavior on a large scale. One of its significant but complex characteristics is its viscosity, which is dependent mainly on temperature, pressure, and stress [5]. In earlier studies of mantle convection, scientists assumed constant viscosity (e.g. [6, 7]) but later, among many others Moresi and Solomatov [8, 9], studied the temperature-dependent viscosity case numerically and concluded that the formation of an immobile lithosphere on terrestrial planets like Mars and Venus seems to be a natural result of temperature-dependent viscosity. However, studies with purely temperature-dependent viscosity cannot portray the true convection

(c) The Author(s) 2022. Open Access This article is licensed under a Creative Commons Attribution 4.0 International License, which permits use, sharing, adaptation, distribution and reproduction in any medium or format, as long as you give appropriate credit to the original author(s) and the source, provide a link to the Creative Commons licence, and indicate if changes were made. The images or other third party material in this article are included in the article's Creative Commons licence, unless indicated otherwise in a credit line to the material. If material is not included in the article's Creative Commons licence and your intended use is not permitted by statutory regulation or exceeds the permitted use, you will need to obtain permission directly from the copyright holder. To view a copy of this licence, visit http:// creativecommons.org/licenses/by/4.0/. 
pattern of the Earth's mantle. As a result, convection with temperature and pressuredependent viscosity is becoming more important, and some notable works in this area have recently been published [10-14]. Christensen [10] showed that additional pressure dependence of viscosity strongly influences the flow regimes. In a 2D axi-symmetrical model, Shahraki and Schmeling [15] examined the simultaneous effect of pressure and temperature-dependent rheology on convection and geoid above the plumes, and Fowler et al. [16] studied the asymptotic structure of mantle convection at high viscosity contrast.

According to King et al. [17], when pressure increases through the mantle, there is a corresponding increase in density due to self-compression. In a vigorously convecting mantle, the rate at which viscous dissipation, which is the irreversible process that changes other forces into heat, is non-negligible and contributes to the heat energy of the fluid, resulting in adiabatic temperature and density gradients that reduce the vigour of convection. Conrad and Hager [18] proposed that the viscous dissipation and resisting force to plate motion may have significant effects on convection and the thermal evolution history of the Earth's mantle. Leng and Zhong [19] concluded that the dissipation occurring in a subduction zone is $10-20 \%$ of the total dissipation for cases with only temperature-dependent viscosity, whereas Morgan et al. [20] declared that when slabs subduct, about $86 \%$ of the gravitational energy for the whole mantle flow is mostly transformed into heat by viscous dissipation. According to Balachandar et al. [21], numerical simulations of 3D convection with temperature-dependent viscosity and viscous heating at realistic Rayleigh numbers for Earth's mantle reveal that, in the strongly timedependent regime, very intense localized heating takes place along the top portion of descending cold sheets and also at locations where the ascending plume heads impinge at the surface. They also found that the horizontally averaged viscous dissipation is concentrated at the top of the convecting layer and has a magnitude comparable to that of radioactive heating. King et al. [17] worked on a benchmark for 2-D Cartesian compressible convection in the Earth's mantle where they used steady-state constant and temperature-dependent viscosity cases as well as time-dependent constant viscosity cases. In their work, the Rayleigh numbers are near $10^{6}$ and dissipation numbers are between 0 and 2, and they conclude that the most unstable wavelengths of compressible convection are smaller than those of incompressible convection. As the research on mantle convection is growing, the importance of studying viscous dissipation is also increasing since it was suggested that the bending of long and highly viscous plates at subduction zones dissipates most of the energy that drives mantle convection [22]. Some notable recent works on numerical studies of convection and effects of variable viscosity and viscous dissipation have been done by Ushachew et al. [23], Megahed [24], Ferdows et al. [25], Ahmed et al. [26], Fetecau et al. [27].

Although mantle convection is a $3 \mathrm{D}$ problem, many $2 \mathrm{D}$ codes have been developed to gain an understanding of the fundamental mechanism and to minimize the computational cost and complexity. As the Earth's mantle has been affected by many complexities, its basic understanding has been constructed through research on simple Rayleigh-Bénard convection [2]. Over the years, the Rayleigh-Bénard convection has become a benchmark problem in computational geophysics as a paradigm for convection in the Earth's mantle. Although Rayleigh-Benard convection with viscosity variation 
is a well-known topic for mantle convection, very high viscosity variation (up to $10^{30}$ ) for mantle convection is not widely covered. To the best of our knowledge, mantle convection with strongly variable viscosity, which is temperature dependent and also both temperature and pressure dependent with the inclusion of viscous dissipation, has not been studied so far. The governing equation in two-dimensional form ensures the conservation of mass, momentum, and energy and the thermodynamic equation of state. In this study, incompressible mantle convection will be considered where the mantle viscosity depends strongly on both temperature and pressure, and viscous dissipation is also considered. The convection will be investigated at a high Rayleigh number with high viscosity variations across the mantle.

In "Methods" section the full governing equations for mantle convection and the appropriate boundary conditions for classical Rayleigh-Bénard convection in a $2 \mathrm{D}$ square cell are described. The equations are non-dimensionalized and the dimensionless parameters are identified. Though the variable viscosity is defined in an Arrhenius form, a modified form of viscosity is used to improve the efficiency of numerical computation. The computational method for simulation is also described, and the code is verified using some benchmark values. Then the governing model is solved numerically in a unit aspect-ratio cell for extremely large viscosity variations, and steady solutions for temperature and streamlines are obtained. The numerical and graphical results of the computation are described in "Result and discussion" section. Finally, in "Conclusion" section some concluding remarks on the results are given.

\section{Methods}

\section{Governing equations}

A classical Rayleigh-Bénard convection in a two-dimensional unit aspect ratio cell with a free slip boundary condition is taken into account. The temperature difference is fixed between the horizontal boundaries. The convective cell is assumed to be a section of a periodic structure in the associated infinite horizontal layer. When adopting Cartesian coordinates $(x, z)$ with horizontal $x$-axis and vertical $z$-axis, the Boussinesq approximation is assumed, which suggests that density variation is barely vital within the buoyancy term of the momentum equation, so that mass conservation takes the shape of the incompressibility condition [16]. The inertia terms within the Navier-Stokes equations (taking the limit of an infinite Prandtl number) are neglected as well. According to Solomatov [28], the integral viscous dissipation within the layer is often balanced by the integral mechanical work done by thermal convection per unit time, and if the viscosity contrast is large, dissipation in the cold boundary layer becomes comparable with the dissipation in the internal region. Thus, in order to balance the energy equation, the extended Boussinesq approximation is used. Here, "extended Boussinesq approximation" means that apart from the driving buoyancy forces, the fluid is treated as being incompressible all over. The non-Boussinesq effects of the adiabatic gradient and frictional heating are introduced into the energy equation [29]. The governing equations ensure the conservation of mass, momentum, and energy. This also ensures a suitable thermodynamic equation of state. The Navier-Stokes equations, which describe the motion in component forms, are [30] 


$$
\begin{aligned}
\frac{\partial u}{\partial x}+\frac{\partial w}{\partial z} & =0, \\
\frac{\partial P}{\partial x} & =\frac{\partial \tau_{1}}{\partial x}+\frac{\partial \tau_{3}}{\partial z}, \\
\frac{\partial P}{\partial z} & =\frac{\partial \tau_{3}}{\partial x}-\frac{\partial \tau_{1}}{\partial z}-\rho g, \\
\tau_{1} & =2 \eta \frac{\partial u}{\partial x}, \\
\tau_{3} & =\eta\left(\frac{\partial u}{\partial z}+\frac{\partial w}{\partial x}\right), \\
\rho & =\rho_{0}\left[1-\alpha\left(T-T_{b}\right)\right],
\end{aligned}
$$

The energy equation is

$$
\frac{\partial T}{\partial t}+\boldsymbol{u} \cdot \nabla T-\frac{\alpha T}{\rho C_{\mathrm{p}}}\left(\frac{\partial P}{\partial t}+\boldsymbol{u} \cdot \nabla P\right)=\kappa\left(\nabla^{2} T\right)+\frac{\tau^{2}}{2 \eta \rho C_{\mathrm{p}}} .
$$

Here, $P$ is the pressure, $\tau$ is the deviatoric stress tensor, $t$ is time, $\rho$ is the density, $\boldsymbol{u}=(u, 0, w)$ is the fluid velocity, where $u$ and $w$ are velocity components in the $x$-and $z$-directions, $g$ is the assumed constant gravitational acceleration acting downwards (the variation of $g$ across the mantle is quite small that it is taken as constant), $\tau_{1}$ and $\tau_{3}$ are the longitudinal and shear components of the deviatoric stress tensor, respectively, $\eta$ is the viscosity, $T_{\mathrm{b}}$ is the basal temperature, $\rho_{0}$ is the basal density, $\kappa$ is the thermal diffusivity, $T$ is the absolute temperature, $C_{\mathrm{p}}$ is the specific heat at constant pressure, and $\alpha$ is the thermal expansion coefficient.

The deviatoric stress tensor, $\boldsymbol{\tau}$ can be expressed as

$$
\boldsymbol{\tau}=\tau_{1}^{2}+\tau_{3}^{2}
$$

where $\tau_{1}$ and $\tau_{3}$ are the longitudinal and shear components of the deviatoric stress tensor, respectively.

The Arrhenius form of viscosity function is

$$
\eta=\frac{1}{2 A\left(\tau_{1}^{2}+\tau_{3}^{2}\right)^{\frac{n-1}{2}}} \exp \left[\frac{E+p V}{R T}\right],
$$

where $A$ is the rate factor, $n$ is the flow index, $E$ is the activation energy, $V$ is the activation volume, and $R$ is the universal gas constant [5].

A unit aspect-ratio cell with a free-slip boundary condition is considered. The temperatures at the bottom and top boundaries are taken as constant, and thermal insulation is assumed on the side walls. The boundary conditions are

$$
\begin{aligned}
& w=0, \quad \tau_{3}=0, \quad=T_{\mathrm{b}} \quad \text { on } \quad z=0, \\
& w=0, \quad \tau_{3}=0, \quad T=T_{\mathrm{s}} \quad \text { on } \quad z=d, \\
& u=0, \quad \tau_{3}=0, \quad \frac{\partial T}{\partial x}=0 \quad \text { on } \quad x=0, d .
\end{aligned}
$$

where $d$ is the depth of the convection cell, $T_{\mathrm{b}}$ and $T_{\mathrm{S}}$ are the basal and top temperatures, respectively (Fig. 1). 


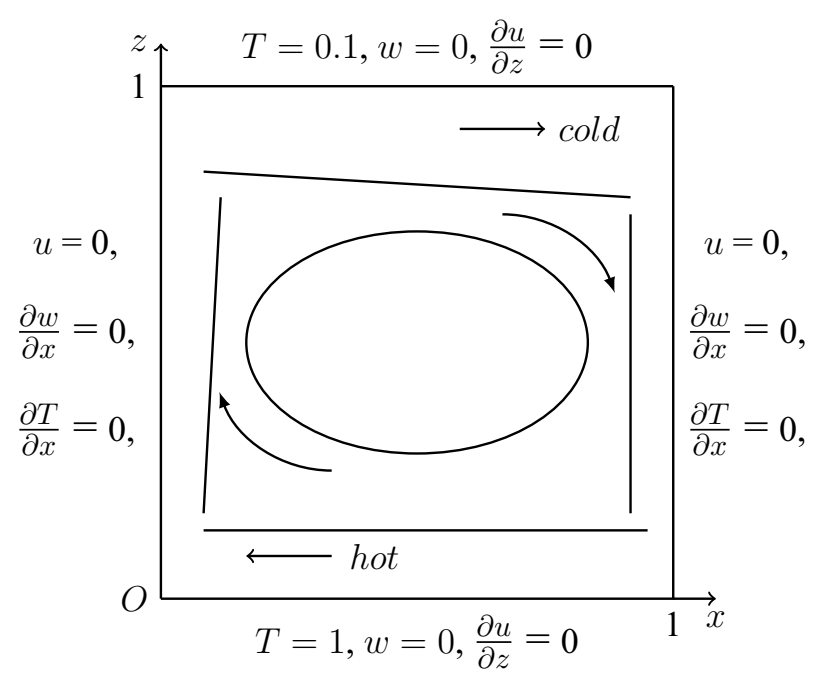

Fig. 1 Schematic diagram of a basally heated non-dimensional unit aspect-ratio cell in mantle

Throughout this work, Newtonian rheology is considered with $n=1$ in the viscosity relation and internal heating is neglected. To see the effects of variable viscosity (both temperature-dependent and temperature-and pressure-dependent viscosity) and viscous dissipation on convection, these assumptions are made to make the model less complicated.

\section{Non-dimensionalization and simplification}

In order to non-dimensionalize the model, the variables are set as $[7,30]$

$$
\begin{array}{r}
\boldsymbol{u}=\frac{\kappa}{d} \boldsymbol{u}^{*}, \quad(x, z)=d\left(x^{*}, z^{*}\right), \quad \boldsymbol{\tau}=\frac{\eta_{0} \kappa}{d^{2}} \boldsymbol{\tau}^{*}, \quad \eta=\frac{e^{(1+\mu) / \varepsilon}}{2 A} \eta^{*}=\eta_{0} \eta^{*}, \\
P=\rho_{0} g d\left(1-z^{*}\right)+\frac{\eta_{0} \kappa}{d^{2}} P^{*}, \quad \rho=\rho_{0} \rho^{*}, \quad t=\frac{d^{2}}{\kappa} t^{*}, \quad T=T_{\mathrm{b}} T^{*}
\end{array}
$$

Using these in equations from (1) to (3) and dropping the asterisk decorations, the dimensionless equations becomes

$$
\begin{aligned}
& \frac{\partial u}{\partial x}+\frac{\partial w}{\partial z}=0, \\
& \frac{\partial P}{\partial x}=\frac{\partial \tau_{1}}{\partial x}+\frac{\partial \tau_{3}}{\partial z}, \\
& \frac{\partial P}{\partial z}=\frac{\partial \tau_{3}}{\partial x}-\frac{\partial \tau_{1}}{\partial z}-\operatorname{Ra}(1-T), \\
& \tau_{1}=2 \eta \frac{\partial u}{\partial x}, \\
& \tau_{3}=\eta\left(\frac{\partial u}{\partial z}+\frac{\partial w}{\partial x}\right), \\
& \frac{\partial T}{\partial t}+\boldsymbol{u} \cdot \nabla T-D T \frac{\bar{B}}{\operatorname{Ra}} \frac{\partial P}{\partial t}+D T w-D T \frac{\bar{B}}{\operatorname{Ra}} u \cdot \nabla P=\nabla^{2} T+\frac{D}{\operatorname{Ra}} \frac{\tau^{2}}{2 \eta},
\end{aligned}
$$

while the dimensionless version of constitutive relation (3) reads 


$$
\eta=\exp \left[\frac{(1-T)(1+\mu)-\mu z+\mu \bar{B} p / \mathrm{Ra}}{\varepsilon T}\right]
$$

in which the dimensionless parameters are,

Dissipation number, $D=\frac{\alpha g d}{C_{\mathrm{p}}}$,

Viscous temperature parameter, $\varepsilon=\frac{R T_{\mathrm{b}}}{E}$,

Viscous pressure number, $\mu=\frac{\rho_{0} g \mathrm{~d} V}{E}$,

Boussinesq number, $\bar{B}=\alpha T_{\mathrm{b}}$,

Rayleigh number, $\operatorname{Ra}=\frac{\alpha \rho_{0} g T_{\mathrm{b}} d^{3}}{\eta_{0} \kappa}$.

Since this model was developed for the mantle, the typical values of the parameters are given in Table 1 , and it is found that for $\mathrm{Ra}>>1, \bar{B} / \mathrm{Ra}$ can be easily ignored. Therefore, the dimensionless energy equation (7) becomes

$$
\frac{\partial T}{\partial t}+\boldsymbol{u} \cdot \nabla T+D T w=\nabla^{2} T+\frac{D}{\operatorname{Ra}} \frac{\tau^{2}}{2 \eta},
$$

and viscosity relation (8) becomes

Table 1 Typical parameter values for mantle convection with variable viscosity

\begin{tabular}{lll}
\hline Parameter & Symbol & Value \\
\hline Mantle depth & $d$ & $3 \times 10^{6} \mathrm{~m}$ \\
Thermal expansion coefficient & $\alpha$ & $2 \times 10^{-5} \mathrm{~K}^{-1}$ \\
Reference density & $\rho_{0}$ & $4 \times 10^{3} \mathrm{~kg} \mathrm{~m}^{-3}$ \\
Gravitational acceleration & $g$ & $10 \mathrm{~m} \mathrm{~s}^{-2}$ \\
Temperature at the top of the mantle & $T_{\mathrm{s}}$ & $300 \mathrm{~K}$ \\
Temperature at the base of the mantle & $T_{\mathrm{b}}$ & $3000 \mathrm{~K}$ \\
Temperature difference & $\Delta T$ & $2700 \mathrm{~K}^{-1}$ \\
Thermal conductivity & $k$ & $4 \mathrm{~W} \mathrm{~m}^{-1} \mathrm{~K}^{-1}$ \\
Specific heat at constant pressure & $C_{\mathrm{p}}$ & $10^{3} \mathrm{~J} \mathrm{~kg}^{-1} \mathrm{~K}^{-1}$ \\
Activation energy & $E$ & $300-525 \mathrm{~kJ} \mathrm{~mol}^{-1}$ \\
Activation volume & $V$ & $6 \times 10^{-6} \mathrm{~m}^{3} \mathrm{~mol}^{-1}$ \\
Gas law constant & $R$ & $8.31 \mathrm{~J} \mathrm{~mol}^{-1} \mathrm{~K}^{-1}$ \\
Viscous rate constant & $A$ & $10^{5} \mathrm{MPa}^{-1} \mathrm{~s}^{-1}$ \\
Thermal diffusivity & $\kappa$ & $1 \times 10^{-6} \mathrm{~m}^{2} \mathrm{~s}^{-1}$ \\
Rayleigh number & $\mathrm{Ra}$ & $10^{7}-10^{9}$ \\
Viscous temperature parameter & $\varepsilon$ & $0.042-0.083$ \\
Viscous pressure number & $\mu$ & $1.2-2.4$ \\
Boussinesq number & $\bar{B}$ & 0.06 \\
Dimensionless surface temperature & $\theta_{0}$ & 0.1 \\
Dissipation number & $D$ & 0.6 \\
\hline
\end{tabular}




$$
\eta=\exp \left[\frac{1-T+\mu(1-z-T)}{\varepsilon T}\right]
$$

This Eq. (11) is known as full form of Arrhenius viscosity function.

The dimensionless boundary conditions (4) become

$$
\begin{aligned}
& w=0, \quad \tau_{3}=0, \quad T=1 \quad \text { on } \quad z=0, \\
& w=0, \quad \tau_{3}=0, \quad T=\frac{T_{\mathrm{s}}}{T_{\mathrm{b}}}=\theta_{0} \quad \text { on } \quad z=1, \\
& u=0, \quad \tau_{3}=0, \quad \frac{\partial T}{\partial x}=0 \quad \text { on } \quad x=0,1 .
\end{aligned}
$$

The dimensionless model consists of governing Eqs. (6), (10), viscosity relation (11) and boundary conditions (12).

\section{Low temperature cut-off viscosity}

To investigate the convection with extremely high viscosity contrasts in the mantle layer, a low temperature cut-off viscosity function is used. This cut-off viscosity relation helps reduce the computational stiffness while retaining the sensitivity of the viscosity to the changes in temperature and pressure across the mantle. It is a well-established fact that in strongly temperature-dependent viscous convection, most of the viscosity variation occurs in a stagnant lid in which the velocity is essentially zero. Based on this fact, the sub-lid convection field is calculated accurately (but not the stress field) by cutting off the dimensionless viscosity at a sufficiently high value that the lid thickness, which essentially only depends on the interaction of the lid temperature with the underlying convection flow, is unaffected.

The low temperature cut-off viscosity function has the following form

$$
\eta= \begin{cases}\exp [Q / \varepsilon] & \frac{Q}{\varepsilon} \leq \log 10^{r}, \\ 10^{r} & \frac{Q}{\varepsilon}>\log 10^{r}\end{cases}
$$

where

$$
Q=\frac{1-T+\mu(1-z-T)}{T}
$$

and the cut-off viscosity value $10^{r}$ is to be chosen appropriately; in numerical experiments, it is chosen $r=6$. Similar type of Arrhenius law with an imposed cut-off viscosity was applied by Huang et al. [31], Huang and Zhong [32], King [33] and Khaleque et al. [13]. A comparison between full-form viscosity function and cut-off viscosity function is shown in "Comparison with benchmark values and validation" section.

Three useful diagnostic quantities which will be used to characterize are viscosity contrast, Nusselt number and root mean square velocity respectively.

The viscosity contrast $\Delta \eta$ is the ratio between the surface and basal values of the viscosity, defined as

$$
\Delta \eta=\exp \left(\frac{1-\theta_{0}-\mu \theta_{0}}{\varepsilon \theta_{0}}\right)
$$


where $\theta_{0}=\frac{T_{\mathrm{s}}}{T_{\mathrm{b}}}$.

The Nusselt number $\mathrm{Nu}$ is the ratio of the average surface heat flow from the convective solution to the heat flow due to conduction. It is calculated in the present case of a square cell by the dimensionless relation

$$
\mathrm{Nu}=-\frac{1}{\left(1-\theta_{0}\right)} \int_{0}^{1} \frac{\partial T}{\partial z}(x, 1) \mathrm{d} x .
$$

$\mathrm{Nu}$ is equal to unity for conduction and exceeds unity as soon as convection starts.

The vigour of the circulating flow is characterised by the non-dimensional RMS (root mean square) velocity. Here RMS velocity is defined by

$$
V_{\mathrm{rms}}=\left[\int_{0}^{1} \int_{0}^{1}\left(u^{2}+w^{2}\right) \mathrm{d} x \mathrm{~d} z\right]^{1 / 2},
$$

where $u$ is the horizontal component of velocity and $w$ is the vertical component of velocity.

\section{Computational method}

In order to solve the dimensionless governing Eqs. (6), (10), (11) with boundary conditions (12) a finite element method based PDE solver 'COMSOL Multiphysics 5.3' is used. The modules for creeping flow, heat transfer in fluids, and Poisson's equation are chosen based on the physics of the model. Free triangular meshing with some refinement near the boundaries of $200 \times 200$ and COMSOL's "extra fine" setting results in a complete mesh of a total of 18,000 elements. As the basis functions or shape functions, Lagrangian P2-P1 elements for creeping flow are selected, which means the shape functions for the velocity field and pressure are Lagrangian quadratic polynomials and Lagrangian linear polynomials, respectively. Similarly, Lagrangian quadratic elements for both temperature in the heat equation and the stream function in Poisson's equation are chosen. For Lagrange elements, the values of all the variables at the nodes are called degrees of freedom (dof) and in this case, our specific discretization finally produces 153,816 degrees of freedom $\left(N_{\mathrm{dof}}\right)$. The following convergence criterion is applied for all cases:

$$
\left(\frac{1}{N_{\text {dof }}} \sum_{i=1}^{N_{\text {dof }}}\left|E_{i}\right|^{2}\right)^{\frac{1}{2}}<\varepsilon
$$

where $E_{i}$ is the estimated error and $\varepsilon=10^{-6}$. Further details of the method can be found in Zimmerman [34].

\section{Comparison with benchmark values and validation}

The values of Nusselt number $\mathrm{Nu}$ and root mean square velocity $V_{\text {rms }}$ are compared with the benchmark values from Blankenbach et al. [35] ${ }^{a}$ and Koglin Jr et al. [36] ${ }^{b}$ in Table 2 for constant viscosity case. Their values were computed for $R a$ up to $10^{6}$ and $10^{7}$ respectively. From Table 2, it is evident that the agreement is within a very good range. 
Table 2 Comparison of computed Nusselt number Nu and RMS velocity $V_{\text {rms }}$ with benchmark values from Blankenbach et al. [35] ${ }^{\mathrm{a}}$ and Koglin Jr et al. [36] ${ }^{\mathrm{b}}$

\begin{tabular}{llllll}
\hline Ra & $\mathbf{N u}$ & & \multicolumn{2}{l}{$\boldsymbol{V}_{\text {rms }}$} \\
\cline { 2 - 3 } & This work & Benchmark & & This work & Benchmark \\
\hline $10^{4}$ & 4.884409 & $4.884409^{\mathrm{a}}$ & 42.864973 & $42.864947^{\mathrm{a}}$ \\
$10^{5}$ & 10.534113 & $10.534095^{\mathrm{a}}$ & & 193.215527 & $193.21454^{\mathrm{a}}$ \\
$10^{6}$ & 21.972563 & $21.972465^{\mathrm{a}}$ & & 834.004359 & $833.98977^{\mathrm{a}}$ \\
$10^{7}$ & 45.638611 & $45.62^{\mathrm{b}}$ & 3633.932754 & - \\
\hline
\end{tabular}

Table 3 Comparison of Nusselt number, Nu of full-form viscosity function (11) and cut-off viscosity function (13) for $\mu=0.0$ and $\mu=0.5$ at $\operatorname{Ra}=10^{7}$ and $\theta_{0}=0.1$

\begin{tabular}{|c|c|c|c|c|}
\hline \multirow[t]{2}{*}{$\Delta \eta$} & \multicolumn{2}{|c|}{ Full form $\eta$} & \multicolumn{2}{|l|}{ Cut-off $\eta$} \\
\hline & $\mu=0.0$ & $\mu=0.5$ & $\mu=0.0$ & $\mu=0.5$ \\
\hline $10^{10}$ & 6.76217 & 8.06845 & 6.76800 & 8.06845 \\
\hline $10^{15}$ & 5.35744 & 6.98327 & 5.36157 & 6.98490 \\
\hline $10^{20}$ & 4.44652 & 6.29296 & 4.45036 & 6.29310 \\
\hline $10^{25}$ & 3.79703 & 5.79253 & 3.80090 & 5.79442 \\
\hline $10^{30}$ & 3.25274 & 5.39134 & 3.25696 & 5.39304 \\
\hline
\end{tabular}

Then the computation is done with variable viscosity with a high viscosity contrast across the mantle layer. The values of Nusselt number $\mathrm{Nu}$ that are compared in Table 3 are found using the full form viscosity function (11) and the cut-off viscosity function (13) for $\mu=0.5$ and $\mu=0.0$. It should be noted that $\mu=0.0$ indicates temperaturedependent viscosity, whereas $\mu \neq 0$ implies that viscosity depends on both temperature and pressure. From Table 3 it can be seen that the values of Nusselt number, Nu with full form viscosity function and the values of Nusselt number, Nu with cut-off viscosity function are very close, which validates the use of the cut-off viscosity function for numerical computation.

\section{Result and discussion}

After validating the model, the governing Eqs. (6), (10) and (13) with boundary conditions (12) are solved. Throughout the computation, the constants $\theta_{0}=0.1$ and $\mathrm{Ra}=10^{7}$ are used, and the values of the Nusselt number, $\mathrm{Nu}$, and root mean square velocity, $V_{\text {rms }}$ for different dissipation numbers, $D$, pressure dependent parameter $\mu$, and temperature dependent parameter $\varepsilon$ are calculated. By varying $\mu$ and $\varepsilon$, different viscosity contrast is obtained across the mantle layer. The numerical computations with $D=0.3$ and $D=0.6$ at $\operatorname{Ra}=10^{7}$ when $\mu=0.0, \mu=0.5$ and $\mu=1.0$ are performed, and the calculated Nusselt number and the RMS velocity values for high viscosity contrasts from $10^{10}$ to $10^{30}$ are shown in Tables 4 and 5.

Tables 4 and 5 show that for each fixed value of $\mu$ and $D, \mathrm{Nu}$ and $V_{\text {rms }}$ decrease as the viscosity contrast increases (i.e., the temperature dependence parameter decreases) across the mantle. It confirms that at the higher viscosity variation, convection becomes weaker, which can also be seen clearly in the thermal distribution Figs. 2 and 3. Nu and $V_{\text {rms }}$ values also decrease as $D$ increases for every particular value of $\mu$. 
Table 4 Nusselt number Nu computed for $\mu=0.0, \mu=0.5, \mu=1.0$ with different viscous dissipation number $D$ at $R a=10^{7}$ and $\theta_{0}=0.1$

\begin{tabular}{|c|c|c|c|c|c|c|c|c|c|}
\hline \multirow[t]{3}{*}{$\Delta \eta$} & \multicolumn{9}{|c|}{ Nusselt number $\mathrm{Nu}$} \\
\hline & \multicolumn{3}{|l|}{$\mu=0.0$} & \multicolumn{3}{|l|}{$\mu=0.5$} & \multicolumn{3}{|l|}{$\mu=1.0$} \\
\hline & $D=0.0$ & $D=0.3$ & $D=0.6$ & $D=0.0$ & $D=0.3$ & $D=0.6$ & $D=0.0$ & $D=0.3$ & $D=0.6$ \\
\hline $10^{10}$ & 6.76800 & 3.83912 & 2.22500 & 8.06845 & 4.46772 & 2.45665 & 9.35884 & 5.22977 & 2.76453 \\
\hline $10^{15}$ & 5.36157 & 2.90745 & 1.73657 & 6.98490 & 3.62823 & 1.93308 & 8.20184 & 4.58926 & 2.25912 \\
\hline $10^{20}$ & 4.45036 & 2.34275 & 1.48655 & 6.29310 & 3.09495 & 1.63913 & 6.85055 & 4.18656 & 1.93551 \\
\hline $10^{25}$ & 3.80090 & 2.00320 & 1.34157 & 5.79442 & 2.71655 & 1.45780 & 5.43699 & 3.88971 & 1.71162 \\
\hline $10^{30}$ & 3.25696 & 1.77292 & 1.24780 & 5.39304 & 2.40512 & 1.33803 & 4.79113 & 3.64133 & 1.54933 \\
\hline
\end{tabular}

Table 5 RMS velocity $V_{\text {rms }}$ computed for $\mu=0.0, \mu=0.5, \mu=1.0$ with different viscous dissipation number $D$ at $R a=10^{7}$ and $\theta_{0}=0.1$

\begin{tabular}{|c|c|c|c|c|c|c|c|c|c|}
\hline \multirow[t]{3}{*}{$\Delta \eta$} & \multicolumn{9}{|c|}{ RMS velocity $V_{\text {rms }}$} \\
\hline & \multicolumn{3}{|l|}{$\mu=0.0$} & \multicolumn{3}{|l|}{$\mu=0.5$} & \multicolumn{3}{|l|}{$\mu=1.0$} \\
\hline & $D=0.0$ & $D=0.3$ & $D=0.6$ & $D=0.0$ & $D=0.3$ & $D=0.6$ & $D=0.0$ & $D=0.3$ & $D=0.6$ \\
\hline $10^{10}$ & 753.149 & 450.644 & 244.368 & 1000.932 & 580.307 & 292.445 & 1189.156 & 722.471 & 350.050 \\
\hline $10^{15}$ & 594.920 & 314.292 & 161.594 & 894.191 & 468.522 & 204.168 & 949.421 & 640.405 & 273.675 \\
\hline $10^{20}$ & 483.396 & 222.950 & 115.209 & 806.197 & 388.104 & 149.557 & 585.505 & 562.901 & 214.198 \\
\hline $10^{25}$ & 398.149 & 173.658 & 85.850 & 721.782 & 324.978 & 113.462 & 307.379 & 481.827 & 169.948 \\
\hline $10^{30}$ & 308.093 & 139.281 & 65.259 & 634.734 & 259.877 & 87.966 & 273.349 & 398.414 & 136.766 \\
\hline
\end{tabular}

It is also observed that at a specific viscosity contrast as the pressure dependence parameter $\mu$ is increased, both $\mathrm{Nu}$ and $V_{\text {rms }}$ values increase for a fixed dissipation number $D=0.3$ and $D=0.6$. The reason behind this is that even though $\mu$ is increased, $\varepsilon$ is actually decreased to maintain the fixed viscosity contrast. However, for $D=0.0$, the trend is not that smooth at higher viscosity variations. Comparing the $V_{\mathrm{rms}}$ values between $D=0.0$ and $D=0.3$ at $\mu=1.0$ it can be seen that at high viscosity contrasts, the $V_{\text {rms }}$ values for $D=0.3$ are larger than those for $D=0.0$ which are unlike the other values.

The thermal distribution and stream function contours for $\mu=0.0, \mu=0.5$ and $\mu=$ 1.0 are presented in Figs. 2, 3 and 4.

In Figs. 2 and 3 the thermal distribution of the unit aspect ratio convection cell for the values of $D=0.3$ and $D=0.6$ respectively are presented for different viscosity contrasts. In panel $2 \mathrm{a}, \mathrm{b}$ and $3 \mathrm{a}, \mathrm{b}$, the viscosity depends only on temperature (i.e. $\mu=0.0$ ) and in panel $2 \mathrm{c}, \mathrm{f}$ and $3 \mathrm{c}, \mathrm{f}$, the viscosity depends on both temperature and pressure (i.e. $\mu \neq 0.0$ ). At each plot of the temperature profile, the blue region corresponds to the cooler temperature whereas the red region corresponds to the high temperature.

For $\mu=0.0, \mu=0.5$ and $\mu=1.0$, from Figs. 2 and 3 we see that as viscosity contrast $\Delta \eta$ increases the thickness of the cold thermal boundary layer at the top of the cell. At the lower mantle, which is near the core of the Earth, the boundary is hot as the temperature is very high and this temperature continues to increase as the viscosity contrast gets larger. 


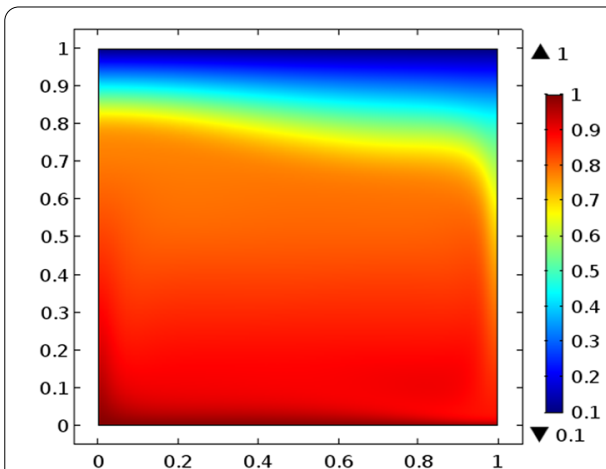

(a) $\Delta \eta=10^{15}, D=0.3, \mu=0.0$.

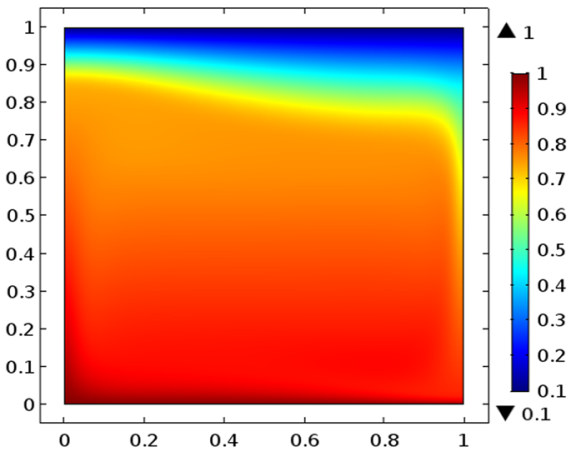

(c) $\Delta \eta=10^{15}, D=0.3, \mu=0.5$.



(e) $\Delta \eta=10^{15}, D=0.3, \mu=1.0$.

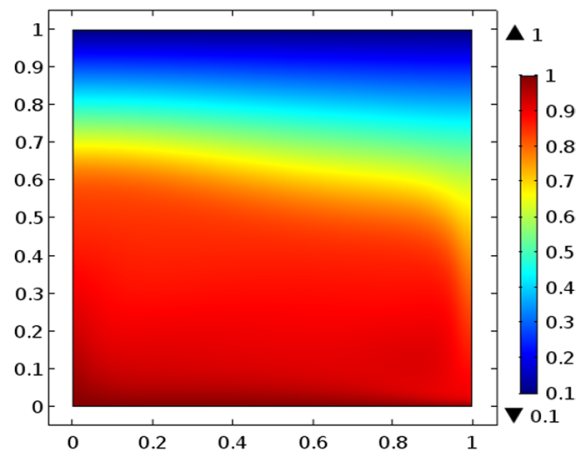

(b) $\Delta \eta=10^{30}, D=0.3, \mu=0.0$.

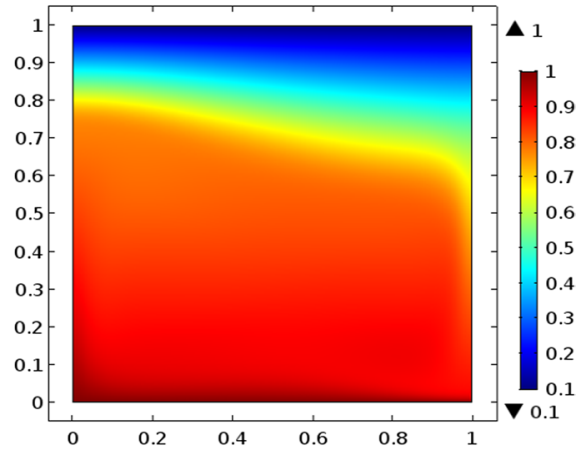

(d) $\Delta \eta=10^{30}, D=0.3, \mu=0.5$.

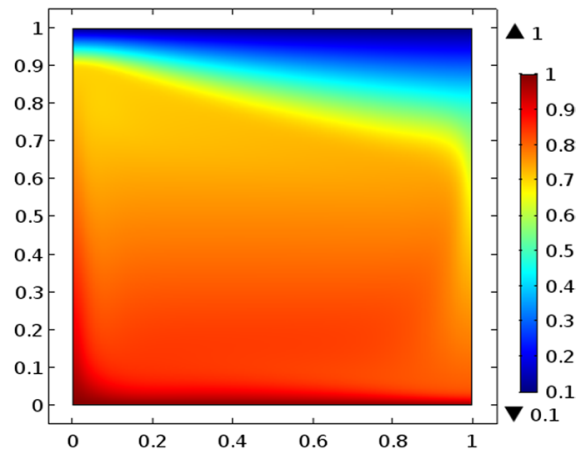

(f) $\Delta \eta=10^{30}, D=0.3, \mu=1.0$.

Fig. 2 Thermal distributions of a convection at different viscosity variations and at different pressure numbers for a fixed viscous dissipation number $D=0.3$ with $\theta_{0}=0.1$ and $\mathrm{Ra}=10^{7}$

The interior temperature decreases significantly as the pressure dependence parameter is included. The convection cell is quite different when viscosity is both temperature and pressure dependent rather than only temperature dependent. Compared to $\mu=0.5$ the significance of pressure can be seen clearly for $\mu=1.0$ from both Figs. 2 and 3.

The stream function contours where stream function $\Psi(x, z)$ defined as

$$
u=-\Psi_{z}, \quad w=\Psi_{x},
$$

are presented in Fig. 4 for $D=0.3$. As the streamlines represent fluid flow, the absence of a streamline confirms that fluid in that region is immobile. In other words, this 


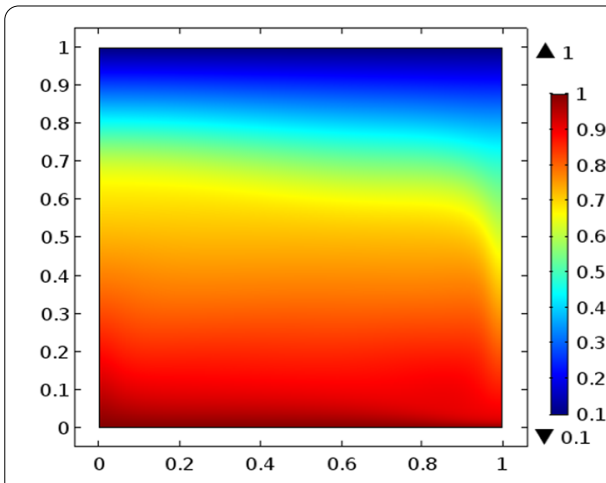

(a) $\Delta \eta=10^{15}, D=0.6, \mu=0.0$.

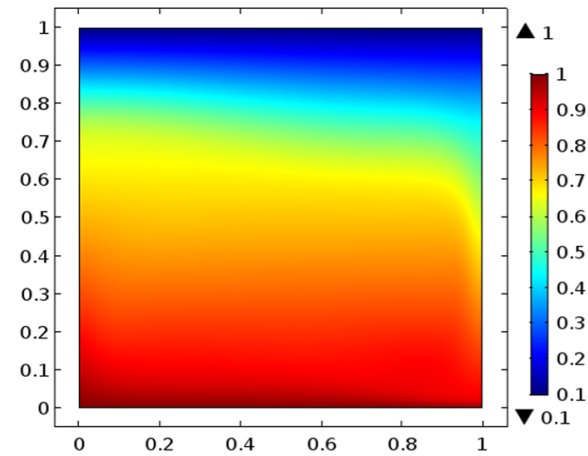

(c) $\Delta \eta=10^{15}, D=0.6, \mu=0.5$.

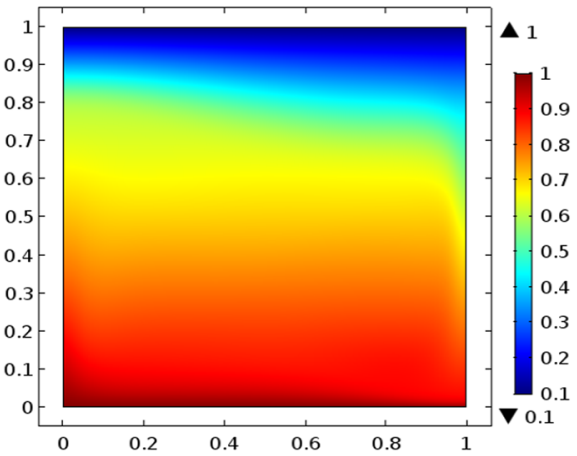

(e) $\Delta \eta=10^{15}, D=0.6, \mu=1.0$.

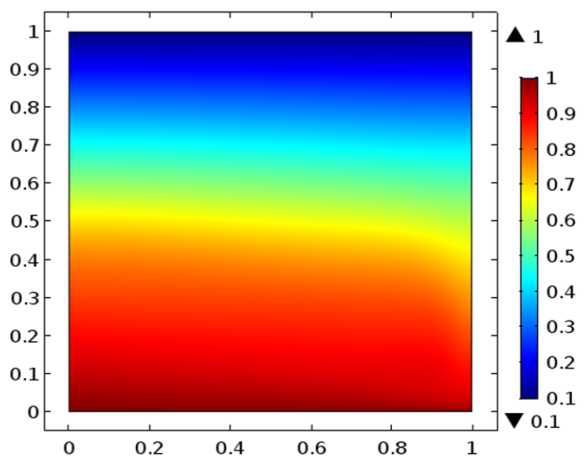

(b) $\Delta \eta=10^{30}, D=0.6, \mu=0.0$.

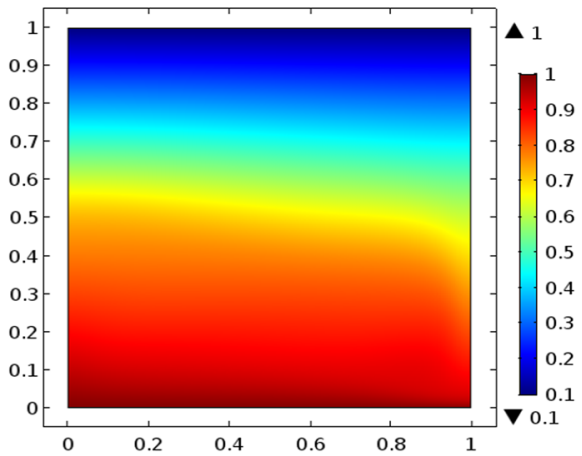

(d) $\Delta \eta=10^{30}, D=0.6, \mu=0.5$.

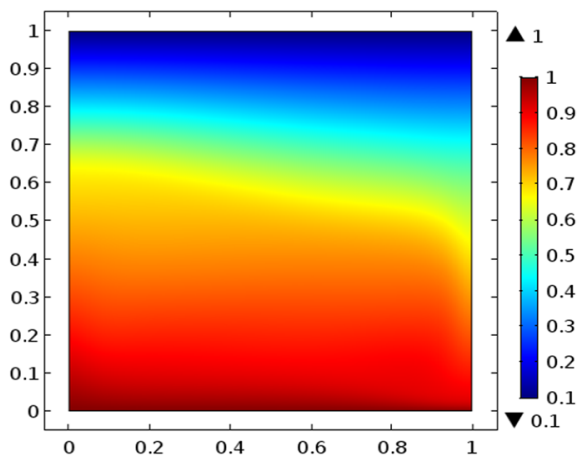

(f) $\Delta \eta=10^{30}, D=0.6, \mu=1.0$.

Fig. 3 Thermal distributions of a convection at different viscosity variations and at different pressure numbers for a fixed viscous dissipation number $D=0.6$ with $\theta_{0}=0.1$ and $\mathrm{Ra}=10^{7}$

immobile region represents the stagnant lid. With increasing viscosity contrast and viscous dissipation, the changes in the convection pattern are very clear. It is observed that the cold thermal boundary layer thickness increases with viscosity contrast. But for a fixed dissipation number, the cold thermal boundary thickness is reduced with the inclusion of the pressure-dependent parameter $\mu$. Clearly, the lid thickness decreases as the pressure dependence parameter is increased at a fixed viscosity variation. However, the lid thickness increases when viscosity variation is increased at a fixed pressure dependence parameter $\mu$ and dissipation number $D$. The Tables 4 and 5 clearly indicate that the heat transfer rate and the root mean square velocity decrease, and Figs. 2, 3 and 4 show that the immobile lid thickness increases as the viscosity contrast at a fixed pressure 


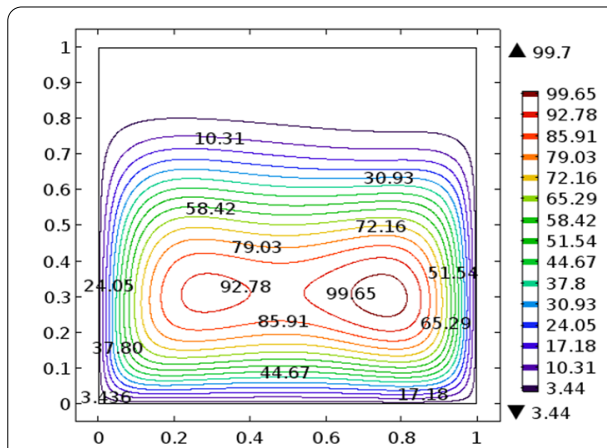

(a) $\Delta \eta=10^{15}, D=0.3, \mu=0.0$.

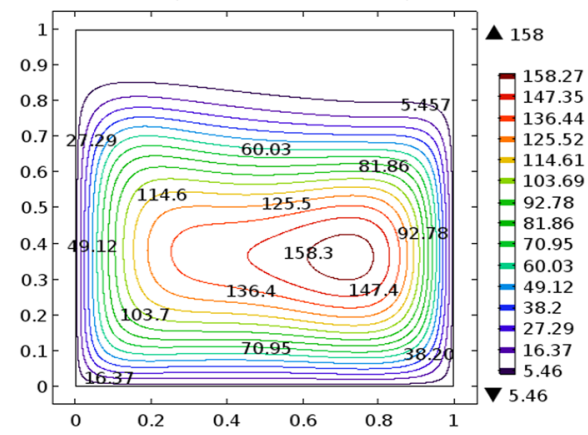

(c) $\Delta \eta=10^{15}, D=0.3, \mu=0.5$.

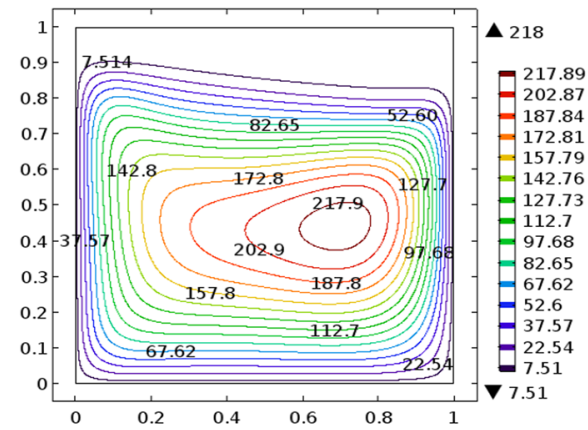

(e) $\Delta \eta=10^{15}, D=0.3, \mu=1.0$.

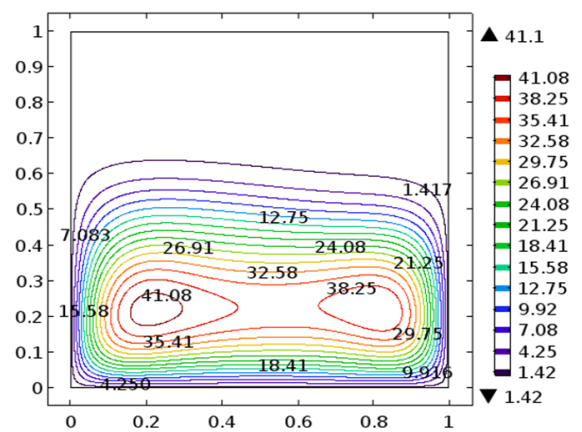

(b) $\Delta \eta=10^{30}, D=0.3, \mu=0.0$.

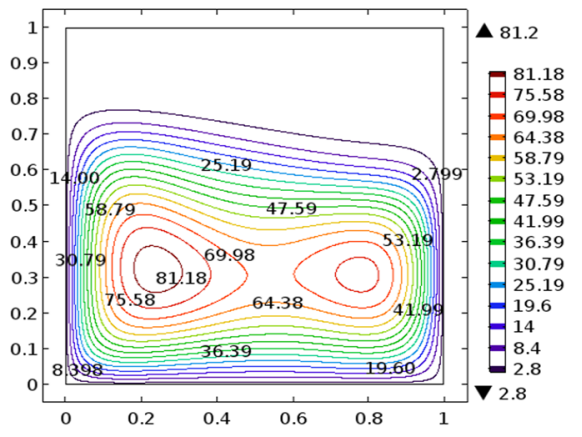

(d) $\Delta \eta=10^{30}, D=0.3, \mu=0.5$.

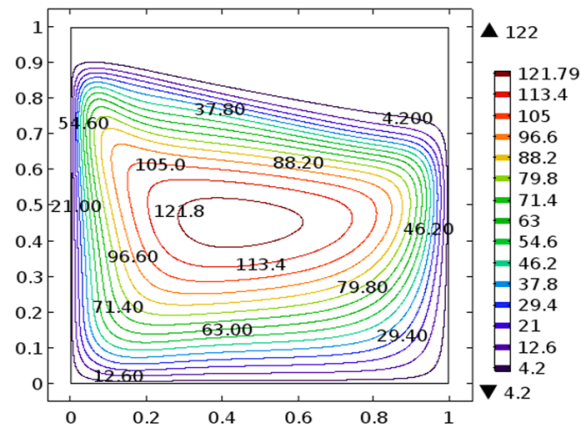

(f) $\Delta \eta=10^{30}, D=0.3, \mu=1.0$.

Fig. 4 Stream function contours of a convection at different viscosity variations and at different pressure numbers for a fixed viscous dissipation number $D=0.3$ with $\theta_{0}=0.1$ and $R a=10^{\prime}$

dependent parameter is increased. The decrease in $\mathrm{Nu}$ and $V_{\mathrm{rms}}$ values, as well as the increase in the thickness of the cold thermal boundary layer, imply that the convection becomes significantly weaker.

A visualization of the isothermal contours in Fig. 5 shows that the hot thermal boundary layer is very thin compared to the cold thermal boundary layer. This figure represents the isothermal contours of a convection cell with temperature dependent viscosity at different viscosity contrast (i.e $\Delta \eta=10^{15}$ and $\Delta \eta=10^{30}$ ) when viscous dissipation numbers are $D=0.3$ and $D=0.5$. There might not be any significant difference in the convection pattern (i.e., isothermal contours), but the contours are not similar. They are clearly affected by different viscous dissipation numbers at different viscosity contrasts. 


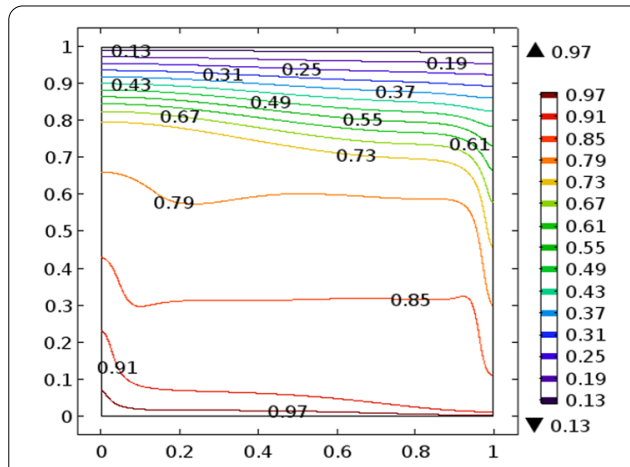

(a) $\Delta \eta=10^{15}, D=0.3$

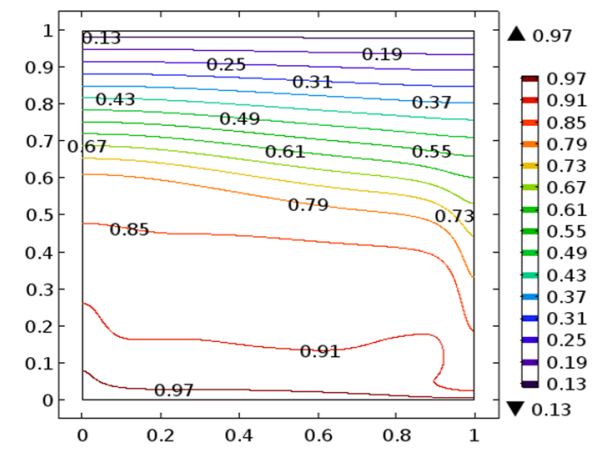

(c) $\Delta \eta=10^{30}, D=0.3$

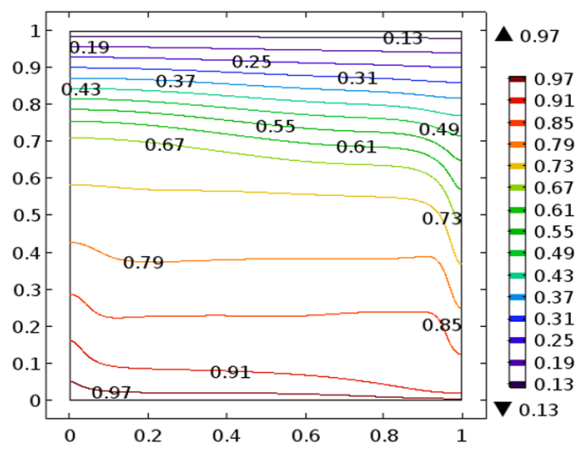

(b) $\Delta \eta=10^{15}, D=0.5$

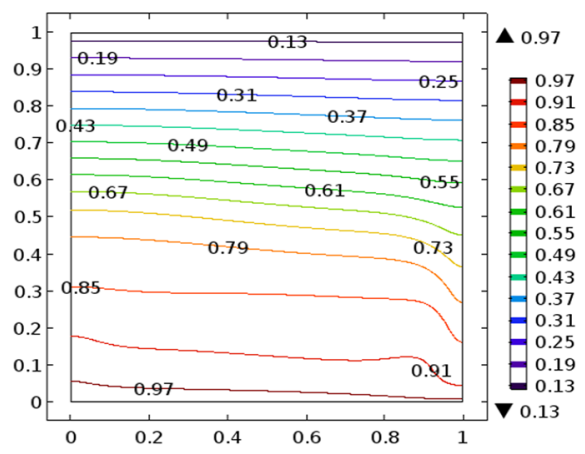

(d) $\Delta \eta=10^{30}, D=0.5$

Fig. 5 Isothermal contours of a temperature dependent viscosity convection at different viscosity variations and viscous dissipation number with $\theta_{0}=0.1$ and $R a=10^{\prime}$

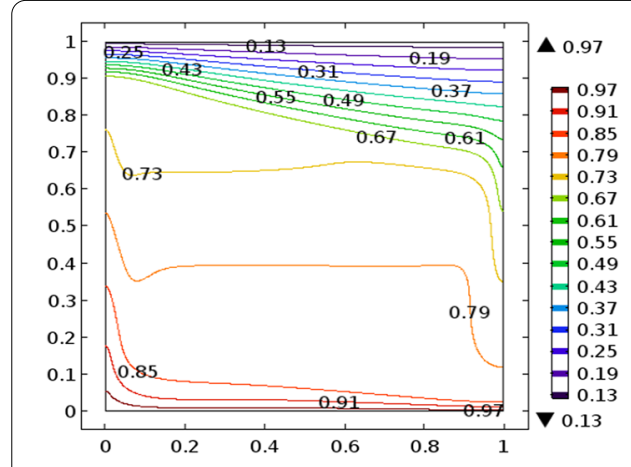

(a) $\Delta \eta=10^{30}, D=0.3, \mu=1.0$.

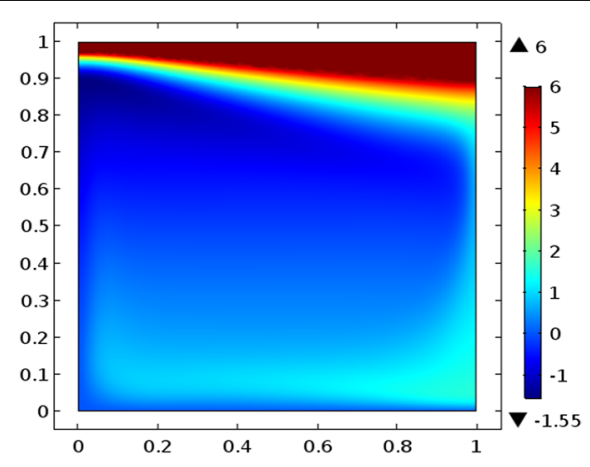

(b) $\Delta \eta=10^{30}, D=0.3, \mu=1.0$.

Fig. 6 a lsothermal contour and $\mathbf{b}$ distribution of $\log _{10} \eta$ for $\mu=1.0$ at $\Delta \eta=10^{30}$ and viscous dissipation $D=0.3$ with $\theta_{0}=0.1$ and $R a=10^{7}$

Isothermal contours (Fig. 6a) and viscosity distribution (Fig. 6b) for $\mu=1.0$ at $\Delta \eta=10^{30}$ and viscous dissipation $D=0.3$ are shown in Fig. 6 . The viscosity variation from top to bottom is shown in Fig. 6b, and the resulting color ranges from the lowest value (blue) to $10^{6}$ (brown). Clearly, the cut-off viscosity function simply ignores the high value of the lid viscosity and considers it as a constant there. Figure $6 \mathrm{~b}$ shows a low viscosity region in the upper mantle and a relatively high viscosity region in the 
lower mantle just above the bottom boundary layer. This implies that the interior is not isoviscous.

Horizontally average temperature vs depth profiles for viscous dissipation of $D=0.3$ and $D=0.6$ are presented in Fig. 7 . These figures show how the horizontally averaged temperature varies with depth at different viscous dissipation numbers and at different viscosity variations. It also shows how it changes for temperature-dependent viscosity and temperature-and pressure-dependent viscosity. The rapid change in temperature near the cold upper boundary and the hot lower boundary explains the strong temperature gradients in those regions. The plots also indicate that the core of the mantle, i.e. the interior, is not isothermal for both the temperature dependent viscosity case and the temperature and pressure dependent viscosity case. The interior of the convection cell undergoes a larger jump in temperature when dissipation effect is stronger $(D=0.6)$. The figures show that the interior temperature increases with the increase of viscosity contrast across the mantle layer for $\mu=0.0$ and $\mu=0.5$ at $D=0.3$ and $D=0.6$. Similar situation occurs for $\mu=1.0$ at $D=0.6$ but when $D=0.3$, temperature decreases at high viscosity contrast (i.e. at $\Delta \eta=10^{30}$ ).

\section{Conclusion}

The study of a basally heated convection model with a strongly temperature and pressure dependent viscous fluid relative to the Earth's mantle in the presence of viscous dissipation has been the principal aim of this work. The classical Rayleigh-Bénard convection model was solved using a low temperature cut-off viscosity function to avoid the stiffness of computation. It was aimed to pursue viscosity that is dependent only on temperature and simultaneously dependent on both temperature and pressure, and a comparison is presented through figures and tables.

According to Jarvis and Mckenzie [37], the dissipation number is between 0.25 and 0.8 , whereas Leng and Zhong [19] estimate $D$ to be 0.5 to 0.7. Ricard [38] found that its value is about 1.0 near the surface, and decreases to about 0.2 near the CMB. From Table $1, D \approx 0.6$ has been found. Thus, the effect of various viscous dissipation numbers for mantle like convection with $\mathrm{Ra}=10^{7}$ is checked. The different values of viscous dissipation number show the changes in heat transfer rate $\mathrm{Nu}$ and root mean square velocity $V_{\text {rms. }}$ It is shown that the fluid is not isothermal and isoviscous in the presence of viscous

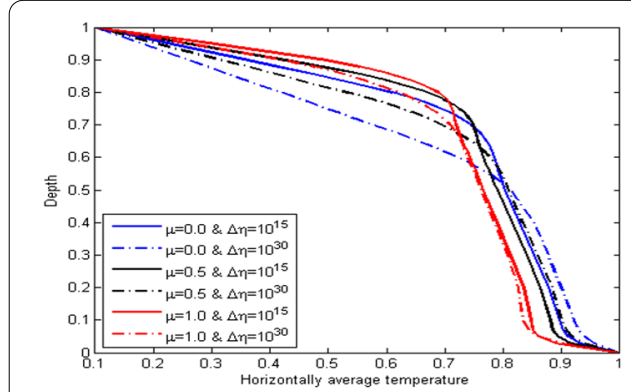

(a) $D=0.3$.

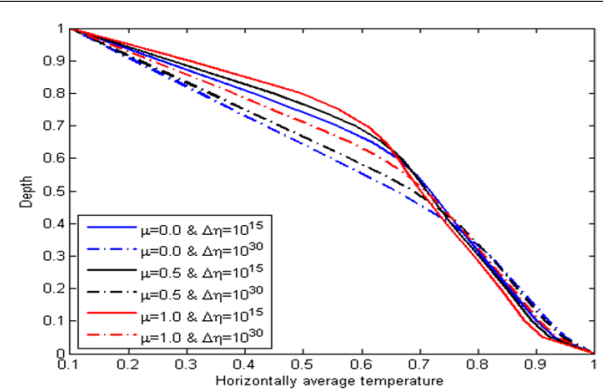

(b) $D=0.6$.

Fig. 7 Horizontally average temperature vs depth profiles at viscosity contrasts $\Delta \eta=10^{15}$ and $\Delta \eta=10^{30}$ for convection with $\mu=0.0, \mu=0.5$ and $\mu=1.0$ at $\theta_{0}=0.1$ and $R a=10^{7}$ with viscous dissipation $D=0.3$ and $D=0.6$ 
dissipation in both cases when viscosity is temperature-dependent and temperaturepressure-dependent. The viscosity distribution at high viscosity contrast for $\mu=1.0$ also showed that the fluid is not isoviscous.

Analysis of the results can predict that if the dissipation number is increased, the lid thickness will increase more and the convection rate will decrease notably. But it is also clear that the inclusion of viscous dissipation does not affect the convection pattern in any drastic way. The convection becomes weaker as viscosity contrast becomes larger and the viscous dissipation number is increased. However, the variation in $\mathrm{Nu}, V_{\mathrm{rms}}$ increase as $\mu$ goes from 0 to 0.5 , but the trend is different when $\mu$ goes from 0.5 to 1.0. Thus, strong pressure dependence in viscosity affects the convection in a different way. For a temperature-dependent viscosity case and a temperature and pressure-dependent viscosity case, the horizontally averaged temperature increases with viscosity contrast in the interior, but the trend is opposite in the top boundary layer, i.e., the stagnant lid. In this study we investigated convection with high viscosity contrast, because for the typical parameter values, it is estimated that the viscosity contrast for the Earth's mantle is $10^{50}$ or more. Without extreme parameter values, it is quite impossible to obtain a proper asymptotic structure of mantle convection for the Earth and other planets. Thus, it is believed that this study will have a significant impact on the study of thermal convection in the Earth's mantle and other planets where viscosity is strongly variable and the variation of the order of magnitude is very large.

\section{Acknowledgements}

The author TS Khaleque's research was partially supported by the University of Dhaka, University Grants Commission, Bangladesh.

\section{Authors' contributions}

SBI and TSK derived the mathematical model and designed the first draft of the manuscript. SBI and SAS carried out the numerical simulations. SBI and TSK provided the literature review and final drafting. This work was carried out in collaboration among all authors. All authors read and approved the final manuscript.

Funding

Not applicable.

Availability of data and materials

The data is generated through a mathematical model solved by numerical simulation.

Declaration

Competing interests

The authors declare that they have no competing interests.

Received: 17 June 2021 Accepted: 13 January 2022

Published online: 09 February 2022

\section{References}

1. Runcorn, S.: Mechanism of plate tectonics: mantle convection currents, plumes, gravity sliding or expansion? Tectonophysics 63, 297-307 (1980)

2. Bercovici, D.: Treatise on Geophysics, Mantle Dynamics, vol. 7. Elsevier, Amsterdam (2010)

3. Karato, S.: Rheology of the Earth's mantle: a historical review. Gondwana Res. 18, 17-45 (2010)

4. Rose, I.R., Korenaga, J.: Mantle rheology and the scaling of bending dissipation in plate tectonics. J. Geophys. Res. Solid Earth 116 (2011). https://doi.org/10.1029/2010JB008004

5. Schubert, G., Turcotte, D.L., Olson, P.: Mantle Convection in the Earth and Planets. Cambridge University Press, Cambridge (2001)

6. Turcotte, D.L., Oxburgh, E.R.: Finite amplitude convective cells and continental drift. J. Fluid Mech. 28, 29-42 (1967). https://doi.org/10.1017/S0022112067001880

7. Jarvis, G.T., Peltier, W.R.: Mantle convection as a boundary layer phenomenon. Geophys. J. Int. 68, 389-427 (1982). https://doi.org/10.1111/j.1365-246X.1982.tb04907.x

8. Moresi, L.N., Solomatov, V.: Numerical investigation of $2 \mathrm{~d}$ convection with extremely large viscosity variations. Phys. Fluids 7, 2154-2162 (1995). https://doi.org/10.1063/1.868465 
9. Solomatov, V., Moresi, L.N.: Three regimes of mantle convection with non-Newtonian viscosity and stagnant lid convection on the terrestrial planets. Geophys. Res. Lett. 24, 1907-1910 (1997). https://doi.org/10.1029/97GL01682

10. Christensen, U.: Convection with pressure- and temperature-dependent non-Newtonian rheology. Geophys. J. Int. 77, 343-384 (1984). https://doi.org/10.1111/j.1365-246X.1984.tb01939.x

11. Doin, M.P., Fleitout, L., Christensen, U.: Mantle convection and stability of depleted and undepleted continental lithosphere. J. Geophys. Res. Solid Earth 77, 2771-2787 (1997)

12. Dumoulin, C., Doin, M.P., Fleitout, L.: Heat transport in stagnant lid convection with temperature- and pressuredependent Newtonian or non-Newtonian rheology. J. Geophys. Res. Solid Earth 104, 12759-12777 (1999)

13. Khaleque, T., Fowler, A., Howell, P., Vynnycky, M.: Numerical studies of thermal convection with temperature- and pressure-dependent viscosity at extreme viscosity contrasts. Phys. Fluids $\mathbf{2 7}, 076603$ (2015)

14. Maurice, M., Tosi, N., Samuel, H., Plesa, A.C., Hüttig, C., Breuer, D.: Onset of solid-state mantle convection and mixing during magma ocean solidification. J. Geophys. Res. Planets 122, 577-598 (2017). https://doi.org/10.1002/2016J E005250

15. Shahraki, M., Schmeling, H.: Plume-induced geoid anomalies from 2D axi-symmetric temperature- and pressuredependent mantle convection models. J. Geodyn. 50-60, 193-206 (2012)

16. Fowler, A.C., Howell, P.D., Khaleque, T.S.: Convection of a fluid with strongly temperature and pressure dependent viscosity. Geophys. Astrophys. Fluid Dyn. 110, 130-165 (2016). https://doi.org/10.1080/03091929.2016.1146264

17. King, S.D., Lee, C., Van Keken, P.E., Leng, W., Zhong, S., Tan, E., Tosi, N., Kameyama, M.C.: A community benchmark for 2-D Cartesian compressible convection in the Earth's mantle. Geophys. J. Int. 180, $73-87$ (2010)

18. Conrad, C.P., Hager, B.H.: The thermal evolution of an earth with strong subduction zones. Geophys. Res. Lett. $\mathbf{2 6}$ 3041-3044 (1999)

19. Leng, W., Zhong, S.: Constraints on viscous dissipation of plate bending from compressible mantle convection. Earth Planet Sci. Lett. 297, 154-164 (2010)

20. Morgan, J.P., Rüpke, L.H., White, W.M.:The current energetics of earth's interior: agravitational energy perspective Front. Earth Sci. 4, 46 (2016)

21. Balachandar, S., Yuen, D., Reuteler, D., Lauer, G.: Viscous dissipation in three-dimensional convection with temperature-dependent viscosity. Science 267, 1150-1153 (1995)

22. Conrad, C., Hager, B.: Effects of plate bending and fault strength at subduction zones on plate dynamics. J. Geophys. Res. 104, 17551-17571 (1999)

23. Ushachew, E.G., Sharma, M.K., Makinde, O.D.: Numerical study of MHD heat convection of nanofluid in an open enclosure with internal heated objects and sinusoidal heated bottom. Comput. Therm. Sci. 13(5), 1-16 (2021)

24. Megahed, A.M.: Williamson fluid flow due to a nonlinearly stretching sheet with viscous dissipation and thermal radiation. J. Egypt Math. Soc. 27, 12 (2019). https://doi.org/10.1186/s42787-019-0016-y

25. Ferdows, M., Murtaza, M.G., Shamshuddin, M.: Effect of internal heat generation on free convective power-law variable temperature past a vertical plate considering exponential variable viscosity and thermal conductivity. J. Egypt Math. Soc. 27, 56 (2019). https://doi.org/10.1186/s42787-019-0062-5

26. Ahmed, Z., Nadeem, S., Saleem, S., Ellahi, R.: Numerical study of unsteady flow and heat transfer CNT-based MHD nanofluid with variable viscosity over a permeable shrinking surface. Int. J. Numer. Method H 29, 4607-4623 (2019)

27. Fetecau, C., Vieru, D., Abbas, T., Ellahi, R.: Analytical solutions of upper convected Maxwell fluid with exponential

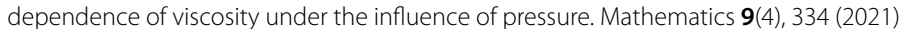

28. Solomatov, V.S.: Scaling of temperature and stress dependent viscosity convection. Phys. Fluids 7, 266-274 (1995). https://doi.org/10.1063/1.868624

29. Christensen, U.R., Yuen, D.A.: Layered convection induced by phase transitions. J. Geophys. Res. Solid Earth 90, 10291-10300 (1985)

30. Fowler, A.: Mathematical Geoscience, vol. 36. Springer, Berlin (2011)

31. Huang, J., Zhong, S., van Hunen, J.: Controls on sublithospheric small-scale convection. J. Geophys. Res. Solid Earth 108 (2003). https://doi.org/10.1029/2003JB002456

32. Huang, J., Zhong, S.: Sublithospheric small-scale convection and its implications for the residual topography at old ocean basins and the plate model. J. Geophys. Res. Solid Earth. 110 (2005). https://doi.org/10.1029/2004JB003153

33. King, S.D.: On topography and geoid from 2-d stagnant lid convection calculations. Geochem. Geophys. Geosyst. 10 (2009) https://doi.org/10.1029/2008GC002250

34. Zimmerman, W.B.: Multiphysics Modeling with Finite Element Methods. World Scientific Publishing Company, Singapore (2006)

35. Blankenbach, B., Busse, F., Christensen, U., Cserepes, L., Gunkel, D., Hansen, U., Harder, H., Jarvis, G., Koch, M., Marquart, G., et al.: A benchmark comparison for mantle convection codes. Geophys. J. Int. 98, 23-38 (1989). https://doi.org/ 10.1111/j.1365-246X.1989.tb05511.x

36. Koglin Jr, D.E., Ghias, S.R., King, S.D., Jarvis, G.T., Lowman, J.P.: Mantle convection with reversing mobile plates: a benchmark study. Geochem. Geophys. Geosyst. 6 (2005). https://doi.org/10.1029/2005GC000924

37. Jarvis, G.T., Mckenzie, D.P.: Convection in a compressible fluid with infinite Prandtl number. J. Fluid Mech. 96 515-583 (1980)

38. Ricard, Y.: Physics of mantle convection. In: Bercovici, D. (ed.) Treatise on Geophysics. 7, 31-87 (2009)

\section{Publisher's Note}

Springer Nature remains neutral with regard to jurisdictional claims in published maps and institutional affiliations. 